\title{
Erratum
}

\section{Reductive Ring Opening of Oxygen-Containing Benzo-Fused Heterocycles by an Arene-Catalysed Lithiation}

Miguel Yus, * Benjamín Moreno, Francisco Foubelo Synthesis 2004, 1115.

In the structure of compounds $\mathbf{5 a}, \mathbf{b}$, both in the Abstract and in the Scheme 1, a methyl group was missed. The correct formulae are as follows:

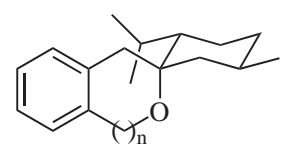

5a $(78 \%)$

$\mathbf{5 b}(55 \%)$

The authors apologize. 